\title{
Investigation into the Impact and Buffering Characteristics of a Non-Newtonian Fluid Damper: Experiment and Simulation
}

\author{
Jingya Sun, Sujuan Jiao, Xiuchang Huang, and Hongxing Hua \\ Institute of Vibration, Shock and Noise, Shanghai Jiao Tong University, No. 800 Dongchuan Road, Shanghai 200240, China \\ Correspondence should be addressed to Hongxing Hua; hhx@sjtu.edu.cn
}

Received 15 March 2014; Revised 30 May 2014; Accepted 30 May 2014; Published 24 June 2014

Academic Editor: Alicia Gonzalez-Buelga

Copyright (C) 2014 Jingya Sun et al. This is an open access article distributed under the Creative Commons Attribution License, which permits unrestricted use, distribution, and reproduction in any medium, provided the original work is properly cited.

\begin{abstract}
Dampers are widely applied to protect devices or human body from severe impact or harmful vibration circumstances. Considering that dampers with low velocity exponent have advantages in energy absorption, they have been widely used in antiseismic structures and shock buffering. Non-Newtonian fluid with strong shear-thinning effect is commonly adopted to achieve this goal. To obtain the damping mechanism and find convenient methods to design the nonlinear fluid damper, in this study, a hydraulic damper is filled with 500,000 cSt silicone oil to achieve a low velocity exponent. Drop hammer test is carried out to experimentally obtain its impact and buffering characteristics. Then a coupling model is built to analyze its damping mechanism, which consists of a model of impact system and a computational fluid dynamics (CFD) model. Results from the coupling model can be consistent with the experiment results. Simulation method can help design non-Newtonian fluid dampers more effectively.
\end{abstract}

\section{Introduction}

To promote working precision or avoid impact-vibration damage, dampers are widely used in vehicles [1-3], civil buildings [4-6], weapons [7-12], spacecraft [13, 14], and so on, to absorb energy of vibrations or shocks and suppress the resonance or alleviate the shock acceleration. Among kinds of dampers, because of high energy absorption capacity, hydraulic damper always appeals to lots of researchers' attention.

To enhance the energy absorption capacity, nonNewtonian fluid with shear-thinning effect is commonly adopted as the flow media of the damper. For most double rod hydraulic dampers, the damping force can be described as

$$
f(t)=c|\dot{\delta}|^{n-1} \dot{\delta}
$$

where $\delta$ is the relative displacement across the damper; $\dot{\delta}$ that represents the derivation of $\delta$ is the relative velocity; $c$ is the damping coefficient; $n$ is the velocity exponent [5, 15-17]. Ruzicka and Derby [15] considered the influence of such velocity- $n$th power damping on vibration isolation, and it is concluded that under the same excitation amplitude, if the velocity exponent is less than unity, the damping can lead to better displacement transmissibility than traditional linear damping. Peng et al. [18], Kovacic et al. [16], and Guo et al. [6] derived similar conclusions with those from Ruzicka and Derby. In buffering applications, to absorb the shock energy in a certain permitted travel, the max damping force should be as small as possible to prevent objects or human from being injured; therefore, in certain conditions dampers with the low velocity exponent are preferred [10].

However, velocity exponent of dampers is determined not only by the shape of damping orifice, but also by Reynolds numbers of the flow through the damping orifice and properties of media fluid. Considering the eddy flow throng the damping orifice will enlarge the velocity exponent, to obtain a damper with low velocity exponent, it is necessary to make the flow through the orifice be laminar. In this condition, the velocity exponent is approximate to the rheological index of the fluid filled in the damper [19]. Therefore, it is also necessary to make the rheological index of the fluid as low as possible. In [19], it is mentioned that high apparent viscosity fluid has a stronger shear-thinning effect, which is helpful to achieve low velocity exponent. In practical applications, 
silicone oil is commonly used as fluid media, because of its nontoxicity and stability. Though in most applications, viscosity of commonly adopted silicone oil is higher than that of hydraulic oil, it is still relatively low. Characteristics of damper with higher viscosity are seldom found in literatures.

Moreover, it is difficult to design a non-Newtonian fluid damper. Formulas derived from Newtonian fluid dynamics are no more suitable. Because non-Newtonian fluid dynamics is abstruse, it is difficult to get a simple and generalized design formula that can guide to make the damper handily. Without a doubt, numerical simulation method is the most efficient way, which supplies a useful tool to predesign the nonNewtonian fluid damper and cut down the product cost. In this study, a damper filled with high viscosity non-Newtonian fluid, 500,000 cSt silicone oil, is manufactured. The damping orifice is of ring gap. Jia et al. [17] derived the semianalytic formula for this kind of damper by considering the flow among the ring gap is purely sheared. However, this flow is of Couette type and the pressure difference cannot be ignored, so the results are untrustful. Hou $[20,21]$ investigated this kind of damper using fluid with 1000 cSt viscosity under the harmonic excitation. The experimental and numerical results can match together but the error is big. This may be due to the inaccurate viscosity and unreasonable numerical method.

In this study, characteristics of a damper with high viscosity silicone oil under a shock condition are investigated. A drop hammer test is carried out to obtain the impact and buffering characteristics of the damper. A coupling model is built to predict the impact and the buffering process. The model consists of the model of impact system and the CFD model of the damper. The supposed model not only can be used to guide the design of the detailed structure such as the size of the damping orifice, but also can supply suggestions in designing the moving parts reasonably.

\section{Structures of the Damper}

The schematic of a non-Newtonian fluid damper is shown in Figure 1. The damper has a two-rod piston which is used to form two symmetrical piston chambers in order to obtain the symmetrical characteristics. The piston stroke is $\pm 55 \mathrm{~mm}$. The diameters of the piston rod and the piston are $8 \mathrm{~mm}$ and $20 \mathrm{~mm}$, respectively. The length of the piston is $20 \mathrm{~mm}$. The inner diameter of the cylinder is $34 \mathrm{~mm}$, so there is an annular gap between the piston and the inner cylinder wall, whose width is $(34-20) / 2=7(\mathrm{~mm})$. The width of the annular gap between the piston rod and the cylinder wall is $(34-8) / 2=13(\mathrm{~mm})$. The piston chambers are fully filled with 500,000 cSt silicone oil.

When an external force is applied to the left piston rod, the piston rod moves toward the right. Silicone oil in the right piston chamber is compressed and flows through the annular gap into the left chamber (as the arrows shown in Figure 1). In this process, damping force is raised to balance the external force. The flow through the ring gap is of typical Couette type, and the damping force consists of two kinds of components. The first is the friction force on the surfaces of the rod and

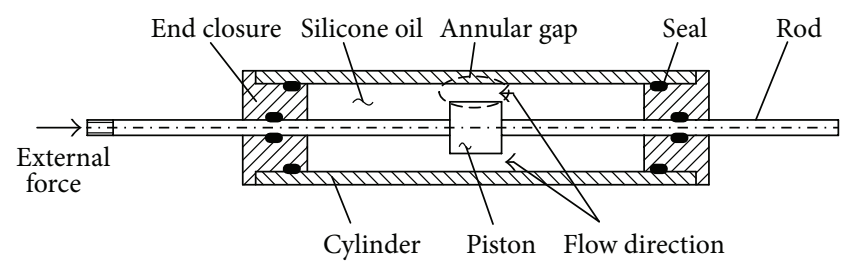

FIGURE 1: Sketch of the non-Newtonian fluid damper.

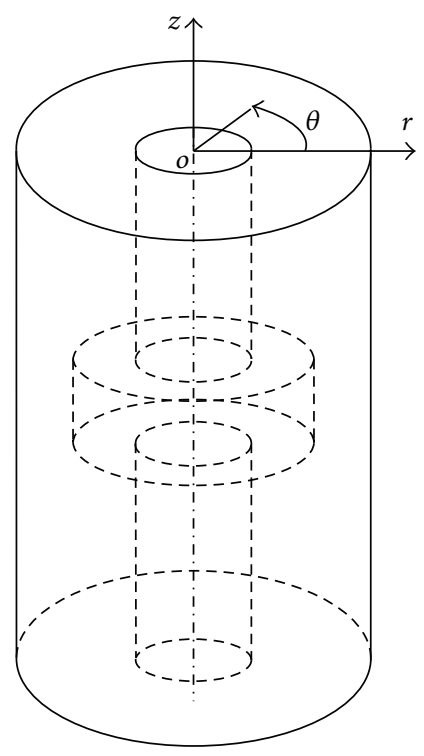

FIGURE 2: Coordinate system for the flow field.

the piston, due to shearing the fluid. The other is from the pressure difference on the piston along the moving axis.

\section{Govern Equations for the Non-Newtonian Fluid}

To describe the govern equations clearly, cylindrical coordinate system is built for the flow field of the damper, seen in Figure 2. Supposing that the fluid is incompressible and its density is constant anywhere, with the flow field being axissymmetric, the flow velocity can be considered as

$$
v_{r}=u(r, z, t), \quad v_{z}=w(r, z, t), \quad v_{\theta}=0 .
$$

The continuity equation and the momentum equation are as follows:

$$
\begin{gathered}
\frac{1}{r} \frac{\partial(r u)}{\partial r}+\frac{\partial w}{\partial z}=0, \\
\rho \frac{d u}{d t}=-\frac{\partial p}{\partial r}+\frac{\partial \tau_{r r}}{\partial r}+\frac{\partial \tau_{r z}}{\partial z}+\frac{\tau_{r r}}{r}, \\
\rho \frac{d w}{d t}=-\frac{\partial p}{\partial z}+\frac{1}{r} \frac{\partial\left(r \tau_{r z}\right)}{\partial r}+\frac{\partial \tau_{z z}}{\partial z},
\end{gathered}
$$

where $\tau_{r r}, \tau_{z z}$, and $\tau_{r z}$ are partial stress components; $p$ is the pressure of the flow field; $\rho$ is the fluid density. 
Because of the strong shear-thinning effect, the viscosity will degrade remarkably as the shear rate is increased. Therefore, the non-Newtonian fluid is commonly considered as the generalized Newtonian fluid which results from a minor modification of the Newtonian fluid constitutive equation [19]. The equations are as follows:

$$
\begin{gathered}
\tau_{r r}=2 \eta(\dot{\gamma}) \frac{\partial u}{\partial r}, \\
\tau_{z z}=2 \eta(\dot{\gamma}) \frac{\partial w}{\partial z}, \\
\tau_{r z}=\eta(\dot{\gamma})\left[\frac{\partial u}{\partial z}+\frac{\partial w}{\partial r}\right],
\end{gathered}
$$

where $\eta(\dot{\gamma})$ is the viscosity function of the shear rate. Many models are supposed to describe the relationship between the viscosity and the shear rate, such as power-law model, Carreau model, Ellis model, and Bingham model. Carreau model can be used to describe the viscosity property of silicone oil $[20,21]$. The model is expressed by

$$
\eta(\dot{\gamma})=\eta_{0}\left[1+(k \dot{\gamma})^{2}\right]^{(n-1) / 2}
$$

where $\eta_{0}$ is the apparent viscosity; $k$ is a parameter which is related to the elastic time constant; $\dot{\gamma}$ is the shear rate.

In this study, the Carreau model is adopted. Parameters in the model are identified according to the measured results, based on the method of least squares. The model with identified parameters is as follows:

$$
\eta(\dot{\gamma})=439.08\left[1+(0.0478 \dot{\gamma})^{2}\right]^{-0.2319} .
$$

Figure 3 shows the relationship between the viscosity and the shear rate. It is clear that the viscosity goes down when being sheared, and the relationship can be well described by Carreau model.

\section{Drop Hammer Test}

The shock characteristics of the damper are tested on a drop machine. Figure 4 shows the sketch of the test rig. The damper is fixed to the foundation. A drop hammer is lifted to a certain height and then falls freely to impact the piston rod of the damper. The damper supplies a damping force to brake the hammer. An acceleration transducer is installed at the top of the drop hammer, whose signal is amplified by an amplifier. A data acquisition system is used to record the acceleration of the drop hammer as it falls. According to the experiment results, the hammer will not rebound after it hits on the piston, so it can be considered that the hammer moves together with the piston in the impact and buffering process.

The lifted height of the drop hammer is from $200 \mathrm{~mm}$ to $1000 \mathrm{~mm}$ with a step $200 \mathrm{~mm}$. The theoretical impact velocity $V_{t}$ when the hammer contacts the piston can be calculated by the following formula:

$$
V_{t}=\sqrt{2 g h}
$$

TABLE 1: Impact velocity of the drop hammer.

\begin{tabular}{lllll}
\hline Drop height $(\mathrm{mm})$ & 400 & 600 & 800 & 1000 \\
\hline Theoretical velocity $(\mathrm{m} / \mathrm{s})$ & 2.80 & 3.43 & 3.95 & 4.43 \\
Impact velocity $(\mathrm{m} / \mathrm{s})$ & 2.75 & 3.32 & 3.74 & 4.30 \\
Error $(\times \%)$ & 1.82 & 3.29 & 5.88 & 2.96 \\
\hline
\end{tabular}

where $g$ is the gravity; $h$ is the drop height. The practical impact velocity $V_{p}$ can be approximately derived by integrating the acceleration. The error of $V_{t}$ and $V_{p}$ is defined as $E_{v}=\left|V_{t}-V_{p}\right| /\left|V_{t}\right|$. All values abovementioned are shown in Table 1. From the table, it is clear that the practical value is in good agreement with the theoretical one and can be used as the initial impact velocity in the following simulation.

\section{Coupling Model for the Shock Buffering Experiment}

5.1. Coupling Model. In the drop hammer test, the whole process can be approximately divided into two parts. The first is the impact process. In this duration, the hammer will collide with the piston and accelerate the moving parts, which mainly includes the rod and the piston, to move at the same speed with itself. In the second process, the hammer and the moving parts will be braked by the damper. The sketch of the whole model is shown in Figure 5(a). In the figure, the model can be considered as two coupling systems. The upper one is the impact system, while the low is the energy absorbing system which only contains a damper.

In the impact and buffering process, the impact system supplies a certain size of velocity to the piston. The damper will feed back damping force to the impact system according to the input velocity. The two systems affect each other by two parameters: the damping force and the velocity of the piston rod. Technical process to simulate the coupling model is described in Figure 5(b).

5.2. Model of Impact System. In Figure 5, $m_{1}$ represents the hammer and $m_{2}$ is the mass of moving parts of the damper such as the piston and its rod. $m_{1}$ and $m_{2}$ connect each other through spring element $k$ and damping element $c_{1}$ which are the stiffness and the damping coefficient in the collision between the drop hammer and the piston rod. $k$ and $c_{1}$ are mainly dominated by the isolation rubber. The whole impact system is supported by a damper.

Based on the supposed model, the differential motion equations of the system are given by

$$
\begin{gathered}
m_{1} \ddot{x}_{1}+k\left(x_{1}-x_{2}\right)+c_{1}\left(\dot{x}_{1}-\dot{x}_{2}\right)=m_{1} g \\
m_{2} \ddot{x}_{2}+k\left(x_{2}-x_{1}\right)+c_{1}\left(\dot{x}_{2}-\dot{x}_{1}\right)+f_{\mathrm{DM}}=m_{2} g
\end{gathered}
$$

where $x_{1}$ and $x_{2}$ are the displacement of $m_{1}$ and $m_{2}$, respectively; $f_{\mathrm{DM}}$ is the output damping force of the damper; $g$ represents the size of the gravity.

In the experiment, the hammer mass is $3.2 \mathrm{~kg}$. The total mass of the piston and its rod is $0.17 \mathrm{~kg}$. The parameters $c_{1}$ and $k$ in the collision can try out approximately based on 


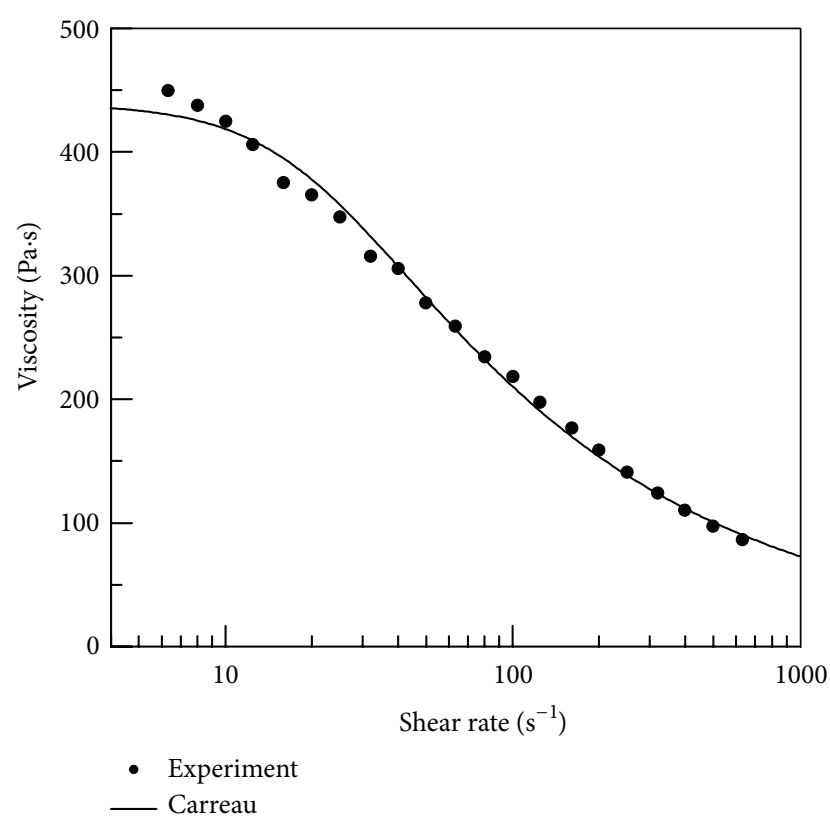

FIGURE 3: Relationship between the viscosity and the shear rate.

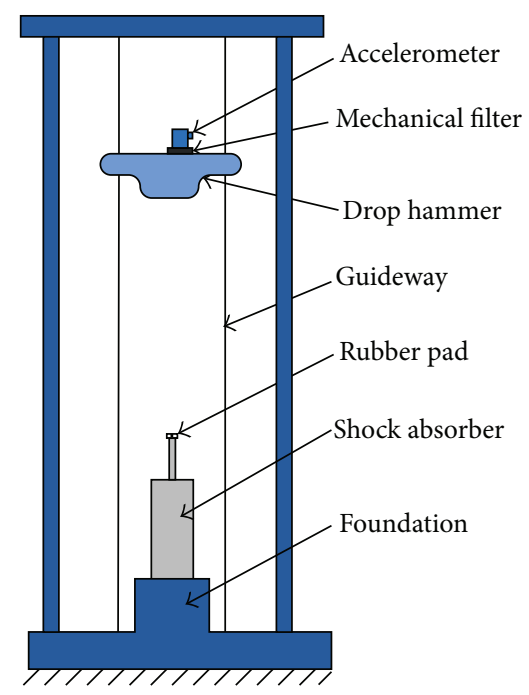

FIGURE 4: Sketch of the test rig.

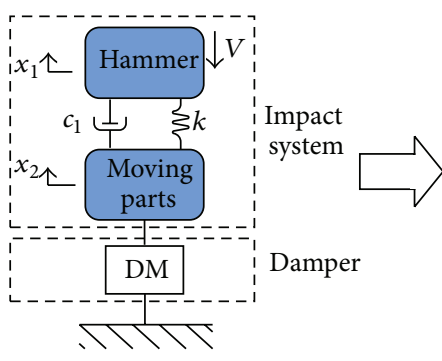

(a)

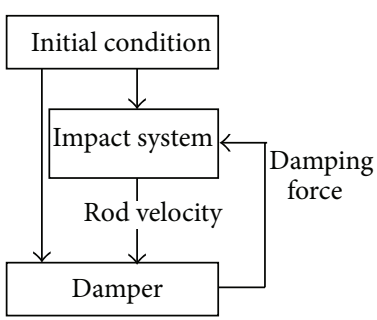

(b)
Figure 5: Model for the shock buffering experiment. (a) Sketch of the coupling model; (b) technical process for coupling numerical simulation.
TABLE 2: Identified parameters of the damper.

\begin{tabular}{ccccccc}
\hline & \multirow{6}{*}{ Parameter } & \multicolumn{6}{c}{ Drop height (mm) } & \multirow{2}{*}{ Averaging } \\
& & 400 & 600 & 800 & 1000 & \\
\hline \multirow{2}{*}{ Experiment } & $n$ & 0.4399 & 0.4524 & 0.4148 & 0.3890 & 0.4240 \\
& $c$ & 476.72 & 442.24 & 443.93 & 426.80 & 447.42 \\
\hline \multirow{2}{*}{ Simulation } & $n$ & 0.5410 & 0.5306 & 0.5245 & 0.5164 & 0.5281 \\
& $c$ & 442.37 & 439.68 & 438.57 & 438.27 & 439.72 \\
\hline
\end{tabular}

the experiment results. The approximate values of $k$ and $c_{1}$ are $k=3.4 \times 10^{6} \mathrm{~N} \cdot \mathrm{m}$ and $c_{1}=400 \mathrm{~N} \cdot \mathrm{s} / \mathrm{m}$, respectively. The initial velocity of $m_{1}$ is set to the practical velocity in Table 2 . The initial velocity of $m_{2}$ is set to zero.

5.3. CFD Model of the Damper. Govern equations (2), (3), and (4) can be solved in CFD software FLUENT, when the flow can be considered as laminar. Viscosity function equation (8) can be inserted into the software program by being compiled as a user defined function. The density of the silicone oil is about $950 \mathrm{~kg} / \mathrm{m}^{3}$.

Due to the axis symmetry characteristic of the damper, fluid zone in Figure 2 can be simplified as shown in Figure 6. The quad elements are used to discretize the computational domain. Meshes are refined at regions where there exists bigger velocity gradient, such as regions close to the piston and the piston rod. The height of the cell close to the piston and the piston rod is $0.2 \mathrm{~mm}$; the height of the others is $0.5 \mathrm{~mm}$. There are 3418 nodes, 6655 faces, and 3238 cells. The nonslip condition is employed in the nodes located at the walls.

Walls of the fluid zone can be divided into two kinds. One is the stationary wall which includes the end surfaces and the cylinder surface, as seen in the blue bold line in Figure 6. In the simulation, these walls will keep stationary; that is, the velocity is set to zero. The other is the moving walls which include surfaces of the rod and piston submerging in fluid, as seen in the red bold line in the figure. In the simulation, the velocity of moving walls is variable. The surfaces must move continuously from time to time. It belongs to a moving boundary problem. Dynamic layering scheme offered by the computed fluid dynamic package FLUENT is used to describe the mesh motion. The mesh is updated at each time step. The piston velocity can be derived from (10),

$$
V_{m_{2}}=\frac{1}{m_{2}} \int_{0}^{t}\left[m_{2} g-k\left(x_{2}-x_{1}\right)-c_{1}\left(\dot{x}_{2}-\dot{x}_{1}\right)-f_{\mathrm{DM}}\right] d t,
$$

where the initial velocity of the piston is set to zero.

During the cosimulation process, in every time step, the output damping force will be extracted from FLUENT solver by a user defined function and feed back to the solver of (10). The solver of (10) will calculate the velocity of piston and feed back to FLUENT. Then in the following time step, the velocity of the piston in the damper will be changed and the output damping force will be got for the next use to calculate the velocity of $m_{1}$ and $m_{2}$. Once the velocity is determined in a time step and transferred to the solver of 


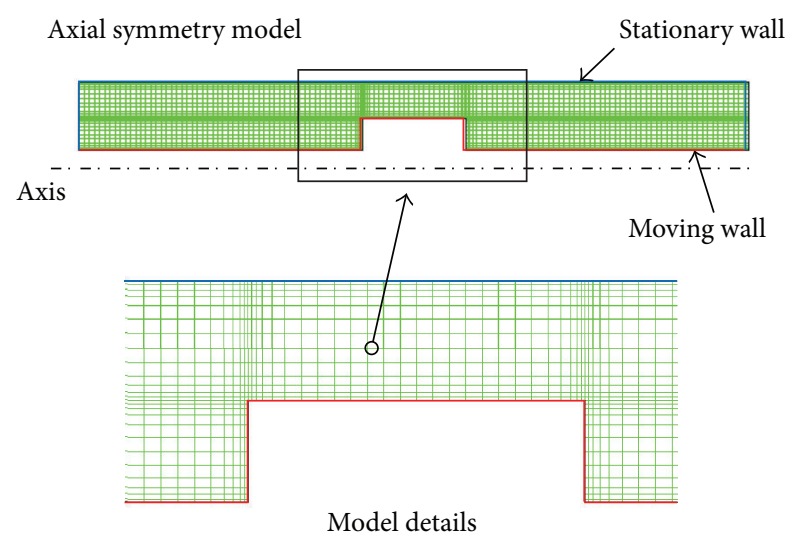

FIGURE 6: Simplified CFD Model of the flow field.

FLUENT, the damping force can be calculated in the next time step. Similarly, the impact and buffering behavior in the whole process can be obtained.

\section{Results and Discussion}

6.1. Experiment Results. Figure 7 shows time history of the shock acceleration of the hammer under different drop heights. It can be found that, in the impact process, the hammer always contacts the piston rod because the size of acceleration is always larger than the gravity. To compare easily, acceleration of the free drop is offset to zero. So the acceleration multiplying the hammer mass represents the output damping force. In the figure, the whole duration lasts from about $20 \mathrm{~ms}$ to $35 \mathrm{~ms}$. It can be divided into two stages, the impact process and the buffering process.

In the impact process, the shock acceleration changes sharply with time, which is mainly due to the collision between the hammer and the piston rod. The piston rod is accelerated to the same velocity of the hammer in an extremely short time. This duration lasts for no more than $2 \mathrm{~ms}$, and the duration seems independent of the drop height. The maximum of the acceleration in this process is remarkably large. From the scaled figure in Figure 7, it is clear to find that, with the drop height increasing, the maximum is approximately increasing linearly, and the rebound acceleration is also reinforced. Many factors can affect this process, such as the mass of the piston and the rod, the mass of the liquid in the damper, and the rubber pad on the top end of the rod.

In the buffering process, the acceleration changes relatively smoothly, but most of the shock energy is absorbed by the damper in this process. Obviously, under different height, the acceleration shape is similar, though the magnitude is different. With the drop height increased, though the magnitude is increasing, the magnitude increment goes down. This is because the silicone oil belongs to non-Newtonian fluid, whose viscosity is shear-thinning. With initial piston velocity increasing, fluid among the damping orifice is sheared more

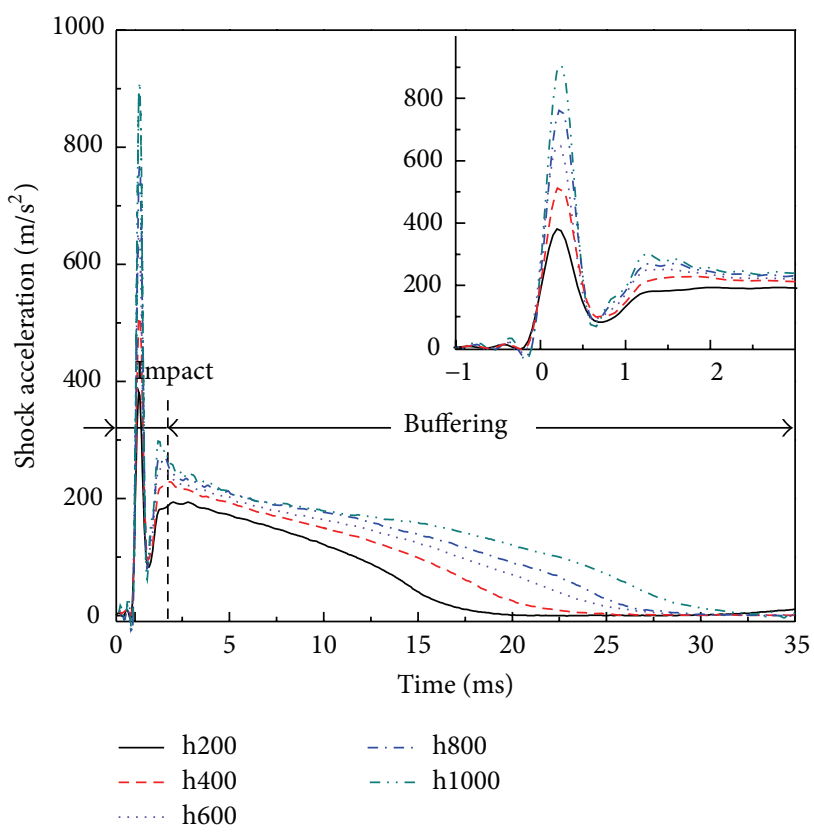

FIgURE 7: Time history of the shock accelerations.

severely, and its viscosity is degraded, which leads to a lower damping force increment.

6.2. Simulation Results and Comparisons. The simulated shock acceleration of the hammer is shown in Figure 8. With comparisons between the simulated and the experimental which are shown in Figure 8, it is easy to find that both results match well with each other. In the impact process, the peak value of the shock acceleration increases as the drop height is increased. The simulated peak value shows the same tendency. The shock duration which seems independent of the drop height also matches well.

In the buffering process, the duration time and the magnitude of the calculated results are approximate to those of the experimental results. Certain level errors still can be observed from the calculated and the experimental results. These errors are caused by the measurement and the simplification in the simplified model.

Generally speaking, the calculated results match well with those from the experimental ones. The supposed coupling model can be used to analyze the influence of parameters on the shock characteristics.

6.3. Analysis on the Output Damping Force. To clearly understand the damping mechanism of the damper, a stress analysis sketch is shown in Figure 9. The output damping force can be divided into three parts. The first part is caused by the pressure differential on the piston $f_{1}=\int_{A_{\text {end }}} p d A$, where $A_{\text {end }}$ is the two end surfaces of the piston. The second part is the friction force on the piston $f_{2}=\int_{A_{\text {cylinder }}} \tau_{\text {piston }} d A$, where $A_{\text {cylinder }}$ is the outer cylinder surface of the piston. The last part is the friction force on the $\operatorname{rod} f_{3}=\int_{A_{\text {rod }}} \tau_{\text {rod }} d A$, where 


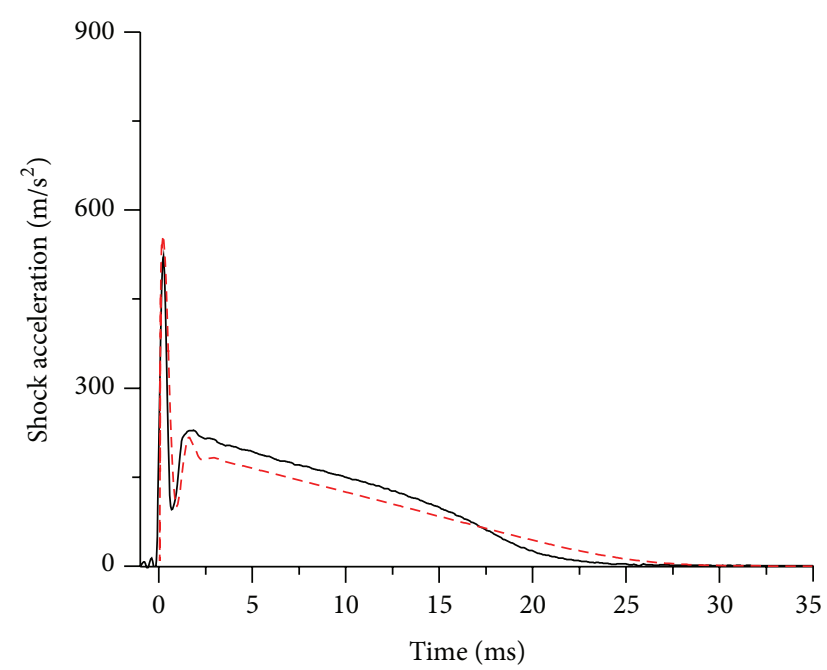

(a)

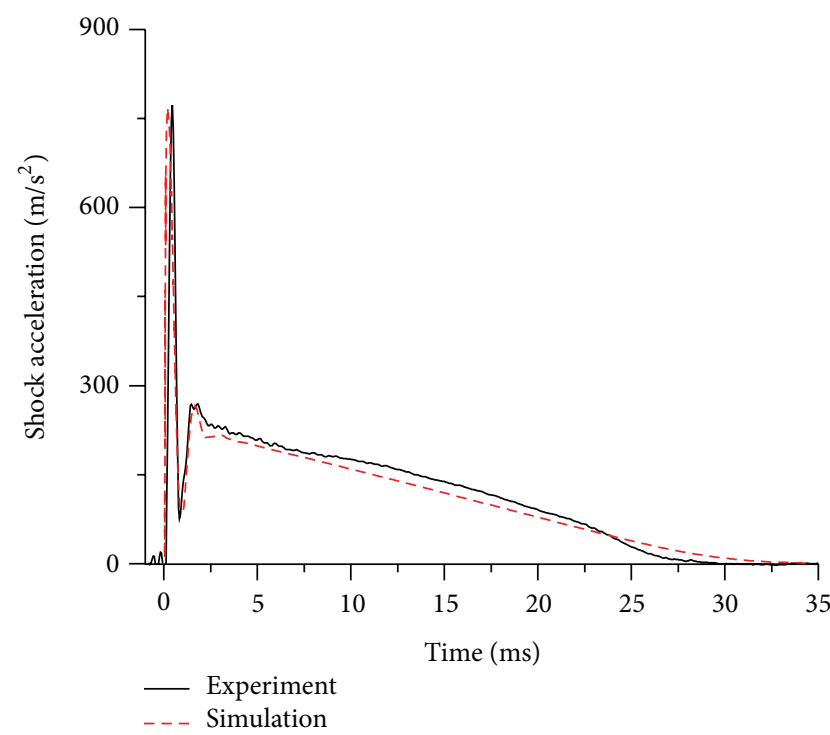

(c)

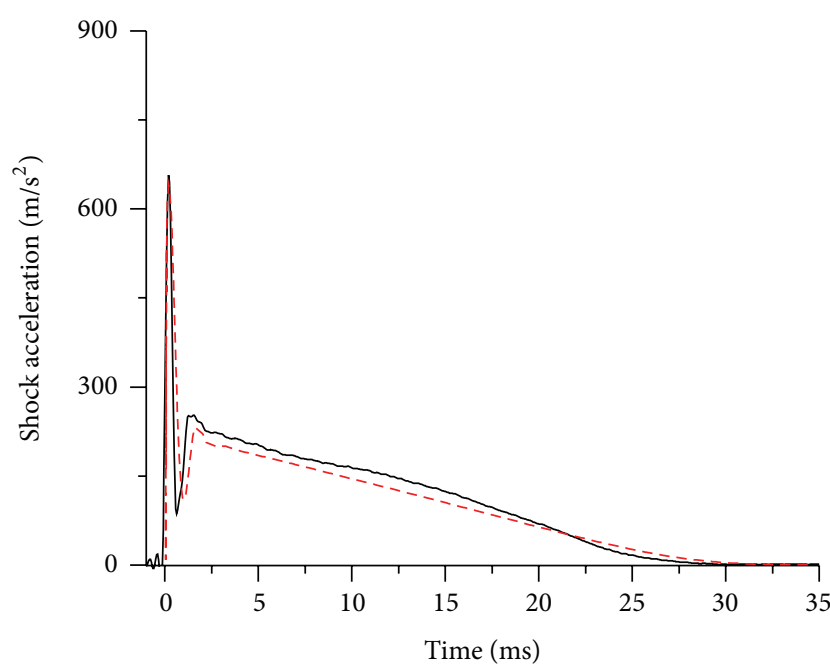

(b)

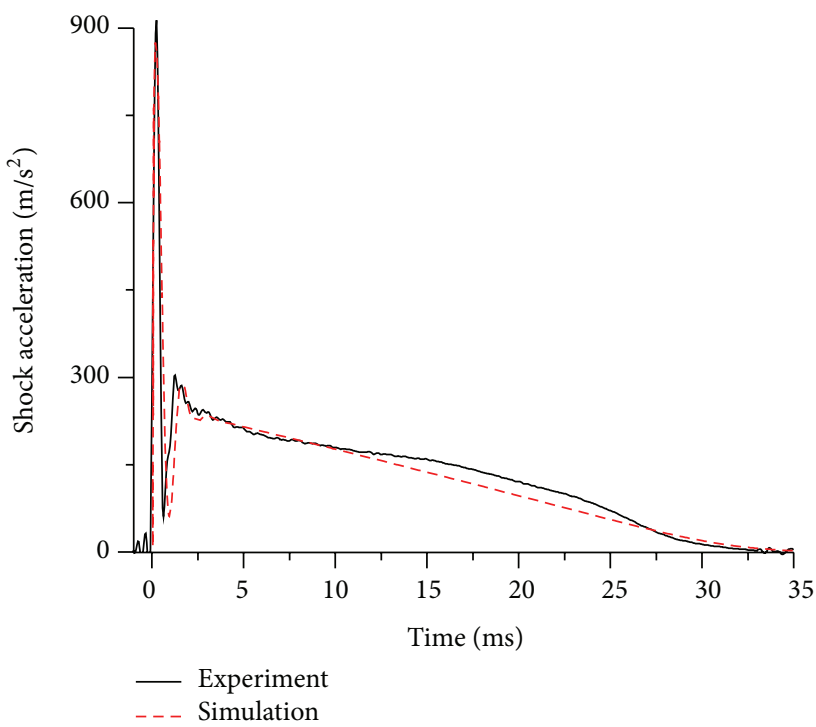

(d)

Figure 8: Comparison between the calculation and the experimental curves. Drop height in (a) $400 \mathrm{~mm}$; (b) $600 \mathrm{~mm}$; (c) $800 \mathrm{~mm}$; (d) $1000 \mathrm{~mm}$. In figures, solid lines represent data from the experiment; dashed lines are from the simulation.

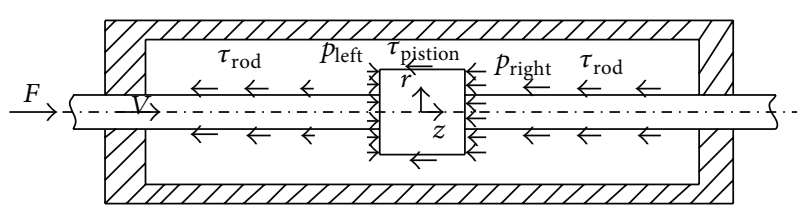

FIGURE 9: Forces imposed on the piston and the rod.

$A_{\text {rod }}$ is the outer cylinder surface of the rod merging in the fluid. So the total output force is $f_{\mathrm{DM}}=f_{1}+f_{2}+f_{3}$.

Figure 10 shows components of the output damping force. Each component nearly keeps the same proportion of the total in the whole process. In the impact process, the force fluctuates obviously. It is mainly caused by coupling behavior between the damper and the hammer. In the following buffering process, the damper force is rather smooth. In this stage, the damping force is supplied to stop the hammer, and most of shock energy is absorbed. From the point of the damping force components, $f_{1}$, the force on the piston due to pressure, is the main component. $f_{2}$ and $f_{3}$, forces on the rod and piston caused by shearing the fluid, also occupy remarkable ratio of the total. This means that, different from the traditional damper filled with low viscosity fluid, it is necessary to consider the shear force on the moving parts for dampers with high viscosity fluid.

To understand the damping characteristics of the damper deeply, (1) is used to describe its mechanics behavior at the buffering process. The identified parameter values from experimental results and simulations are shown in Table 2, while the experimental data at the buffering process is used as the samples. It can be found in the table that, parameters 


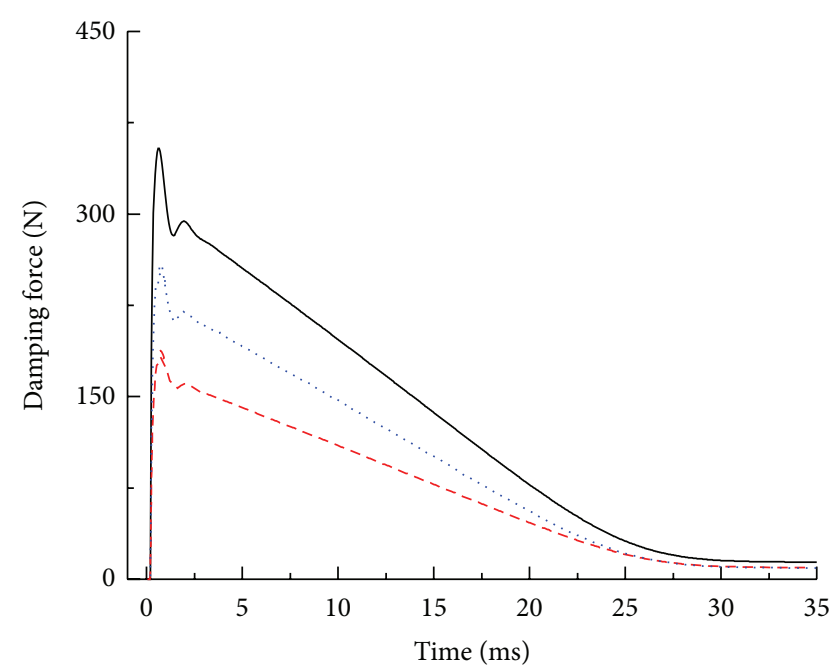

(a)

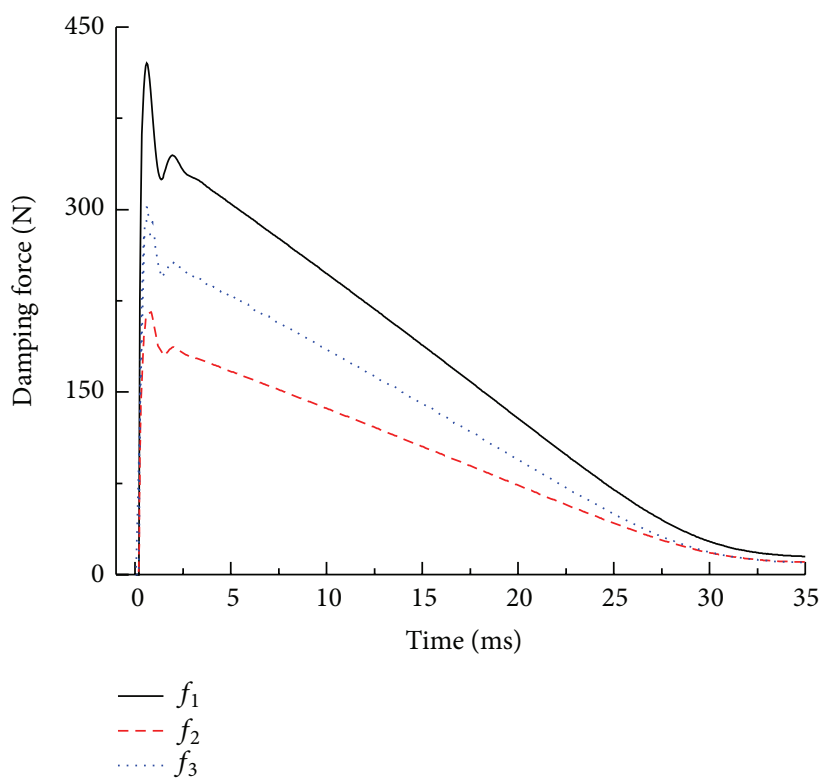

(c)

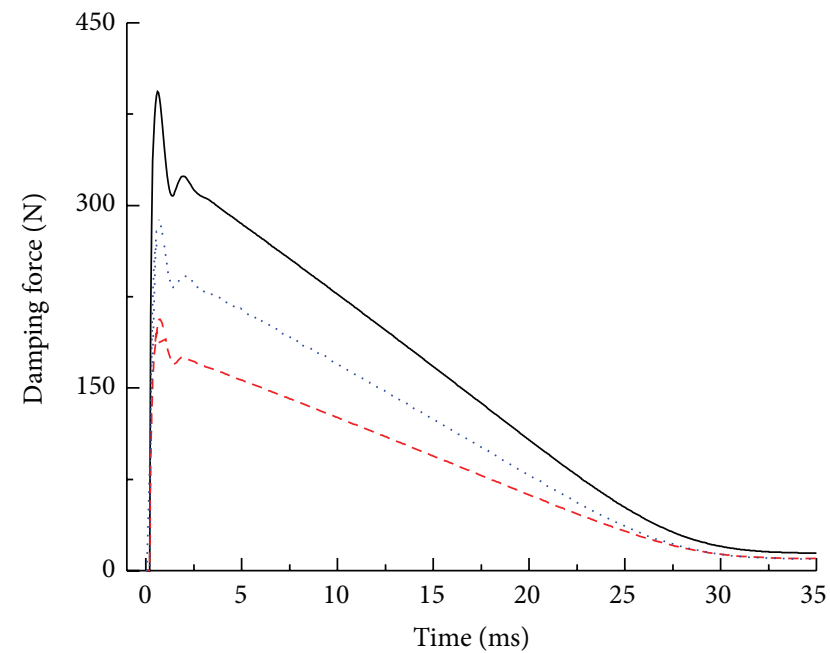

(b)

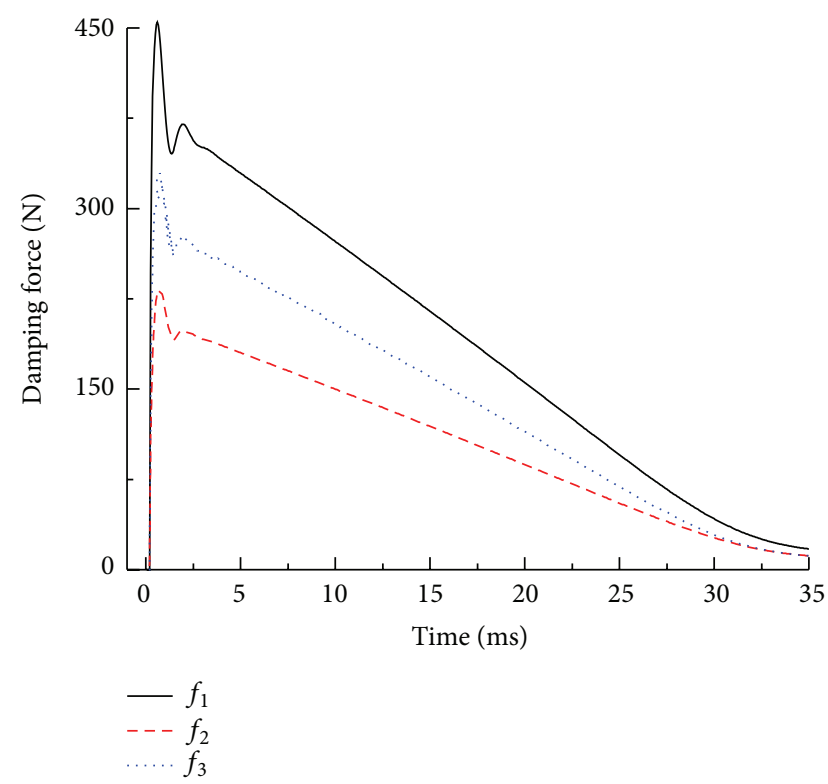

(d)

Figure 10: Components of the damping force from the simulation of drop height $600 \mathrm{~mm}$. In figures, solid line, $f_{1}$; dashed line, $f_{2}$; dotted line, $f_{3}$.

from the experimental and the simulation results match well with each other, although there are some differences. The parameters show a decreasing tendency as the drop height increases.

6.4. Influence of the Mass of the Moving Parts. Mass $m_{2}$ of the moving parts has great effects on the peak of the shock acceleration. To know about the regularity, (10) is solved with different values of $m_{2}$ while the other parameters are kept constant. The corresponding results are shown in Figure 11, where the acceleration curves of $400 \mathrm{~mm}$ drop height are plotted.

Obviously, the influence of $m_{2}$ mainly locates in the impact process. With increasing $m_{2}$, the max acceleration and the overshoot magnitude are increasing. For the shock acceleration, it needs more time to approach the quasi-steady state as $m_{2}$ is increased. So the mass $m_{2}$ should be as small as possible, while the piston and rod can meet the strength and deformation requirements.

6.5. Influence of the Collision Parameters. $k$ and $c_{1}$ are the main collision parameters, which are mainly determined by the isolation rubber at the top of the piston. To analyze the influence of parameters $k, c_{1}$ on the impact process, (10) is solved with different $k, c_{1}$, and $m_{2}$ while the other parameters are kept constant.

In Figure 12, the acceleration curves of drop height $400 \mathrm{~mm}$ with different parameters $k$ and $c_{1}$ are shown. 


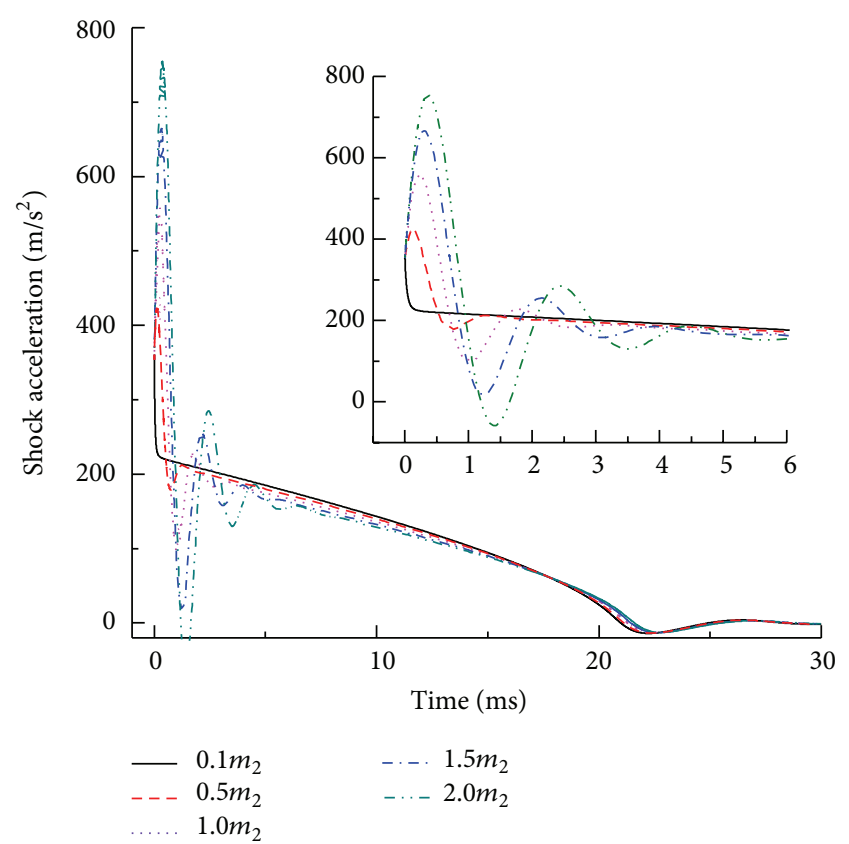

FIGURE 11: Shock acceleration curves from the simplified model with different $m_{2}$ under the drop height $400 \mathrm{~mm}$. In figures, solid line, $0.1 m_{2}$; dashed line, $0.5 m_{2}$; dotted line, $1.0 m_{2}$; dashed-dotted line, $1.5 m_{2}$; dashed-dotted-dotted line, $2.0 m_{2}$.

The influence of $k$ and $c_{1}$ mainly locates in the collision process. The max acceleration as the values of $k$ and $c_{1}$ increases, and the overshoot becomes small. The whole duration will last for a longer time under the small size of $k$ and $c_{1}$, but the buffering process seems to be delayed in a little time. So, in realistic applications, the isolation rubber should be thick enough to reduce the shock magnitude to a certain level.

6.6. Analysis on Flow Fields. In the whole impact and buffering process, the distribution of flow field parameters almost keeps the same except their magnitudes, so we choose the distributions at the simulation time $5 \mathrm{~ms}$ to show its characteristics.

The pressure distribution map is shown in Figure 13. Because the piston moves towards right, pressure in the right chamber is higher than that in the left. The pressure gradient at the piston gap is nearly linear. Near the piston stage, the pressure distribution is disordered. It is easy to find that, in the corner formed by the rod and the end cap, the value is extremely high or low. This is because values near these values are singular, so their values are untractable. However, it is hard to settle because of the shortcoming of the model.

Figure 14 describes the velocity magnitude of the flow field. The velocity of the rod surface is always equal to the same value, while the other walls are stationary. Silicone oil in the right chamber is squeezed through the piston gap into the left. The flow is running towards the left, and the peak value appears at the middle of the gap.

Because of the high viscosity silicone oil with strong shear thinning effect, the viscosity will be degrading with

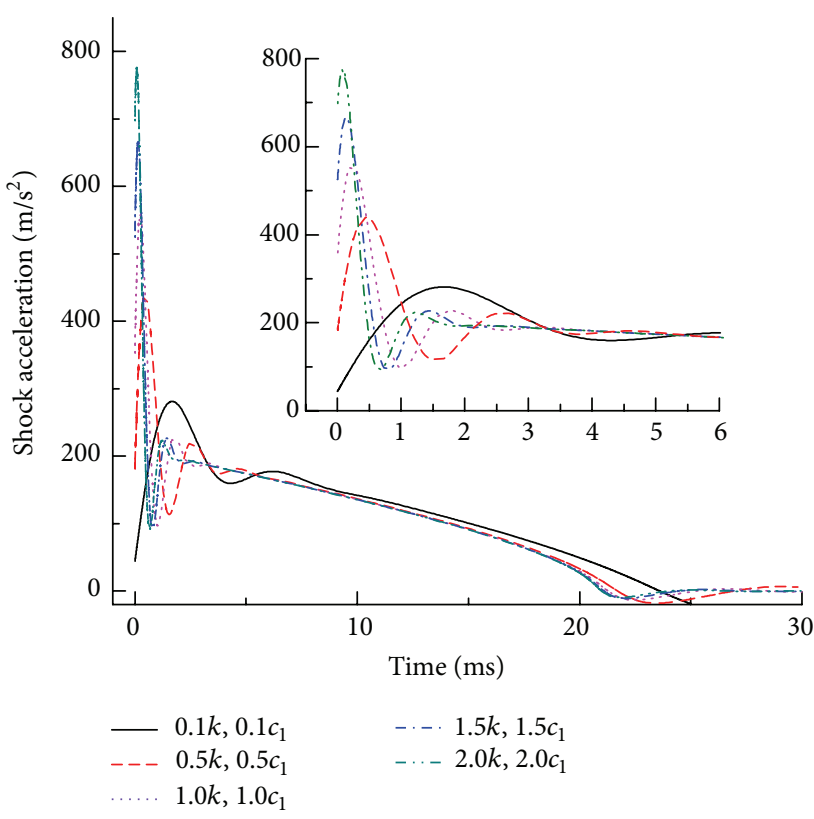

FIGURE 12: Shock acceleration curves from the simplified model with different $k$ and $c_{1}$ under the drop height $400 \mathrm{~mm}$. In figures, solid line, $0.1 m_{2}$; dashed line, $0.5 m_{2}$; dotted line, $1.0 m_{2}$; dashed-dotted line, $1.5 m_{2}$; dashed-dotted-dotted line, $2.0 m_{2}$.

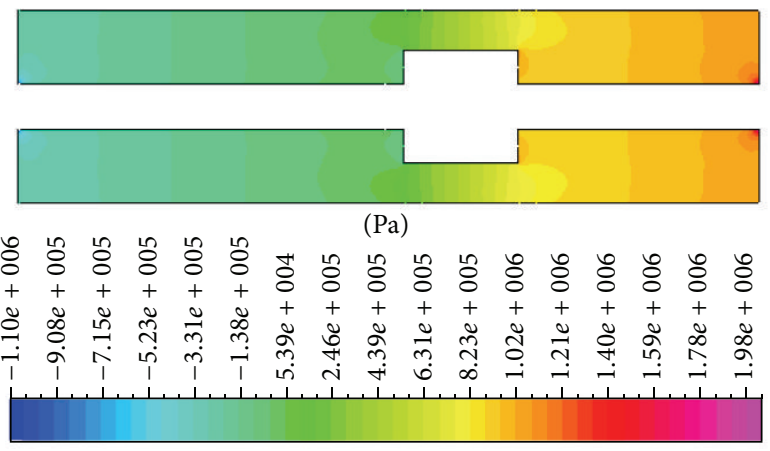

FIgURE 13: Pressure distribution map of the flow field at simulation time $5 \mathrm{~ms}$.
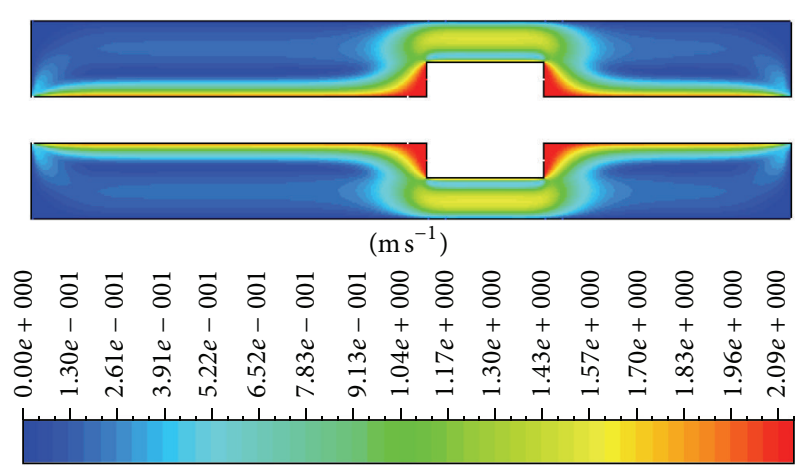

FIGURE 14: Velocity distribution map of the flow field at simulation time $5 \mathrm{~ms}$. 

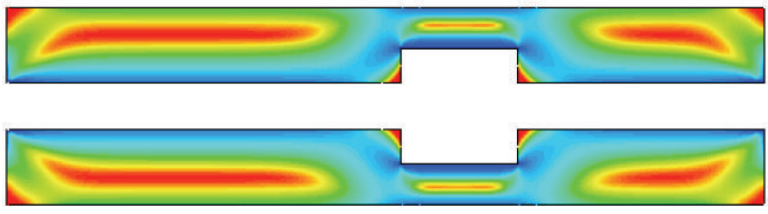

(Pas)

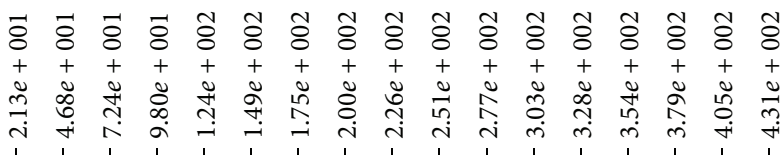

FIGURE 15: Viscosity distribution map of the flow field at simulation time $5 \mathrm{~ms}$.

the velocity gradient increasing. Figure 15 shows the viscosity distributions. This characteristic is different from Newtonian fluid whose viscosity is always constant. In the figure, fluid viscosity near walls is reduced sharply, which means that the fluid shear rate is great. At corners, the fluid is hard to be sheared, so the viscosity keeps its initial value, the apparent viscosity.

The abovementioned three maps, which are helpful to determine which structural details will have main influence on the output damping force, can be used to guide the damper structure design. For example, the manufacture quality at corners will have little effect on the output force.

\section{Conclusions}

In this paper, a non-Newtonian fluid damper filled with 50,0000 cSt silicone oil is made and investigated in a drop hammer test firstly, to get the impact and buffering characteristics experimentally. Then a coupling model is established to predict the shock acceleration and analyze the output damping mechanism. Results from the coupling model can match well with the experiment results. Some conclusions are concluded as follows.

(i) With the drop height being increased, the impact velocity becomes larger. In the impact process, the peak shock acceleration is increasing, and the impact duration is independent of the impact velocity, which is determined by the nature of the impact system. So it is necessary to design the impact system reasonably to reduce the max shock acceleration.

(ii) In different conditions the shock acceleration curves look similar. The buffering characteristic is mainly affected by the velocity exponent.

(iii) From the components of the damping force, it is clear that the friction force occupies a big part of the whole, which is different from the traditional hydraulic damper where the friction force is usually ignored. In design damper with high viscosity fluid, this point should be paid attention. (iv) Through the flow fields, the rule of structures which affected the flow can be summarized easily. It is helpful to design structure details.

Generally speaking, numerical simulation technology is an efficient way to investigate the impact on and buffering characteristics of non-Newtonian fluid damper. And this method is deserved to be used to design high quality fluid damper and cut down research costs for companies.

\section{Conflict of Interests}

The authors declare that there is no conflict of interests regarding the publication of this paper.

\section{Acknowledgment}

The authors gratefully acknowledge the support of the National Science Foundation of China (no. 10872132 and no. 11202128) for this work.

\section{References}

[1] J. Dixon, The Shock Absorber Handbook, John Wiley \& Sons, New York, NY, USA, 2008.

[2] P. Mao, W. Rui-ming, and X. Ming-xiang, "Simulation and experiment study of hydraulic buffer of track vehicle," in Proceedings of the International Conference on Computational Intelligence and Software Engineering (CiSE '09), pp. 1-5, Wuhan, China, December 2009.

[3] F. Wang, M. Tanaka, N. Nakada, T. Hayase, and S. Chonan, "Development of an ER bypass damper for railway car relief couplers," International Journal of Applied Electromagnetics and Mechanics, vol. 19, no. 1-4, pp. 269-274, 2004.

[4] J. Hwang and Y. Huang, "Seismic design of structures with viscous dampers," in International Training Programs For Seismic Design of Building Structures, 2002.

[5] D. Taylor and M. Constantinou, "Testing procedures for high output fluid viscous dampers used in building and bridge structures to dissipate seismic energy," Shock and Vibration, vol. 2, no. 5, pp. 373-381, 1995.

[6] P. Guo, Z. Lang, and Z. Peng, "Analysis and design of the force and displacement transmissibility of nonlinear viscous damper based vibration isolation systems," Nonlinear Dynamics, vol. 67, no. 4, pp. 2671-2687, 2012.

[7] M. Ahmadian, R. Appleton, and J. A. Norris, "An analytical study of fire out of battery using magneto rheological dampers," Shock and Vibration, vol. 9, no. 3, pp. 129-142, 2002.

[8] M. Ahmadian and J. C. Poynor, "An evaluation of magneto rheological dampers for controlling gun recoil dynamics," Shock and Vibration, vol. 8, no. 3-4, pp. 147-155, 2001.

[9] M. Ahmadian, B. Reichert, and X. Song, "System non-linearities induced by skyhook dampers," Shock and Vibration, vol. 8, no. 2, pp. 95-104, 2001.

[10] H. Bao-lin, "Influence of fluid behavior index on performance of a gun recoil magneto-rheological damper," Explosion and Shock Waves, no. 3, pp. 245-249, 2006.

[11] Z. Li and J. Wang, "A gun recoil system employing a magnetorheological fluid damper," Smart Materials and Structures, vol. 21, no. 10, Article ID 105003, 2012. 
[12] K. Li and A. Darby, "An approach to the design of buffer for a buffered impact damper," Structural Control and Health Monitoring, vol. 17, no. 1, pp. 68-82, 2010.

[13] A. Rittweger, J. Albus, E. Hornung, H. Öry, and P. Mourey, "Passive damping devices for aerospace structures," Acta Astronautica, vol. 50, no. 10, pp. 597-608, 2002.

[14] J. Chen, H. Nie, and J.-C. Zhao, "Review of the development of soft-landing buffer for lunar explorations," Journal of Astronautics, vol. 29, no. 3, pp. 731-735, 2008.

[15] J. Ruzicka and T. Derby, Influence of Damping in Vibration Isolation, DTIC Document, 1971.

[16] I. Kovacic, Z. Milovanovic, and M. Brennan, "On the relative and absolute transmissibility of a vibration isolation system subjected to base excitation," Facta Universitatis-Series: Working and Living Enviromental Protection, vol. 5, no. 1, pp. 39-48, 2008.

[17] J. H. Jia, J. Y. Du, Y. Wang, and H. X. Hua, "Design method for fluid viscous dampers," Archive of Applied Mechanics, vol. 78, no. 9, pp. 737-746, 2008.

[18] Z. K. Peng, Z. Q. Lang, and G. Meng, "Evaluation of transmissibility for a class of nonlinear passive vibration isolators," Frontiers of Mechanical Engineering, vol. 7, no. 4, pp. 401-409, 2012.

[19] R. Bird, R. Armstrong, and O. Hassager, Dynamics of Polymeric Liquids, vol. 1 of Fluid Mechanics, 2nd edition, 1987.

[20] C. Hou, "Fluid dynamics and behavior of nonlinear viscous fluid dampers," Journal of Structural Engineering, vol. 134, no. 1, pp. 56-63, 2008.

[21] C. Hou, "Behavior explanation and a new model for nonlinear viscous fluid dampers with a simple annular orifice," Archive of Applied Mechanics, vol. 82, no. 1, pp. 1-12, 2012. 

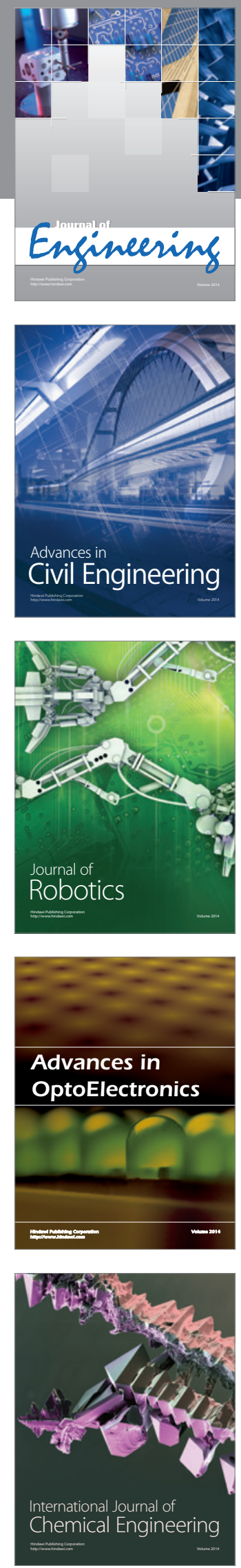

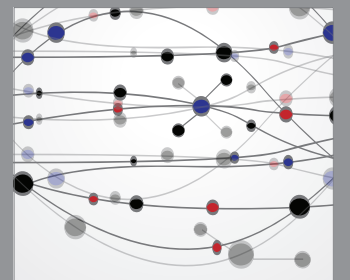

The Scientific World Journal
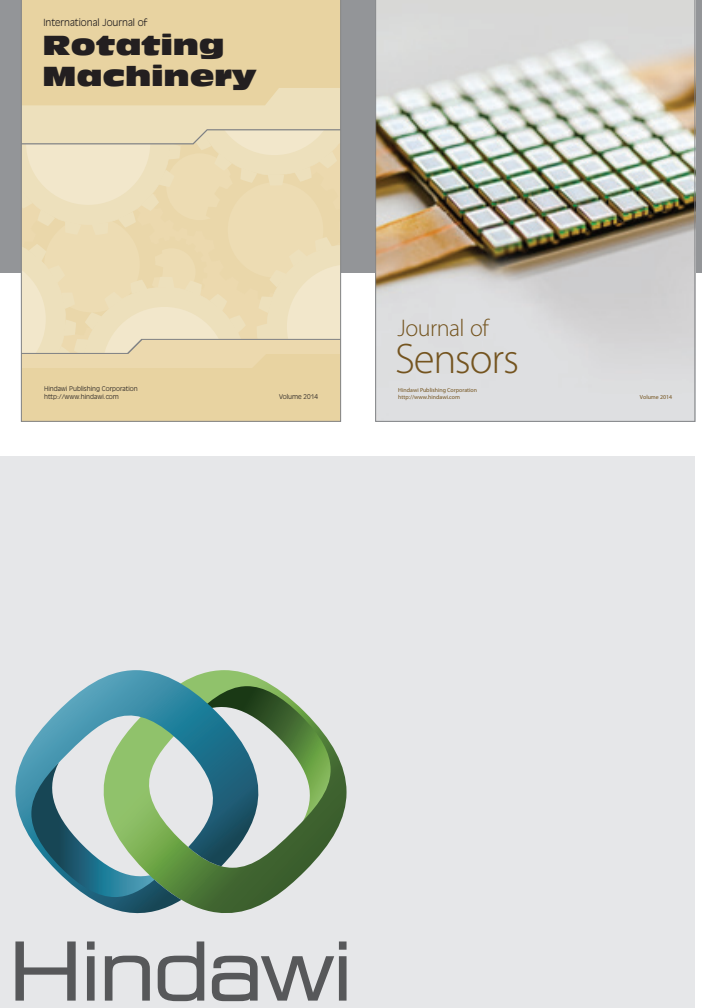

Submit your manuscripts at http://www.hindawi.com
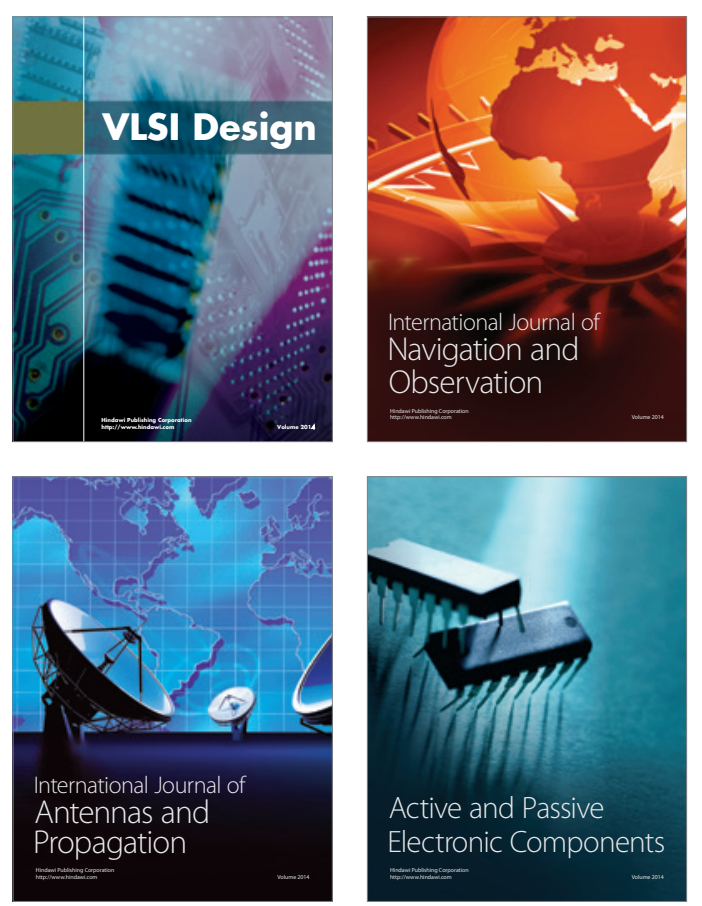
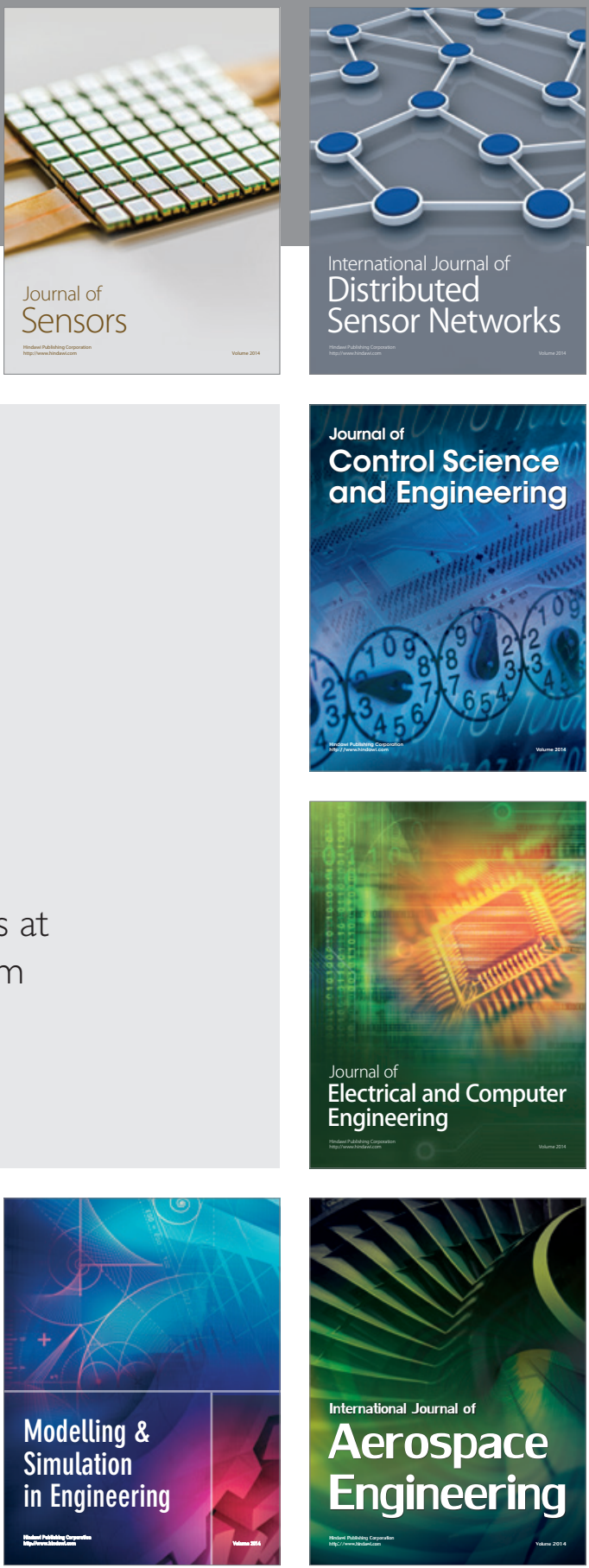

Journal of

Control Science

and Engineering
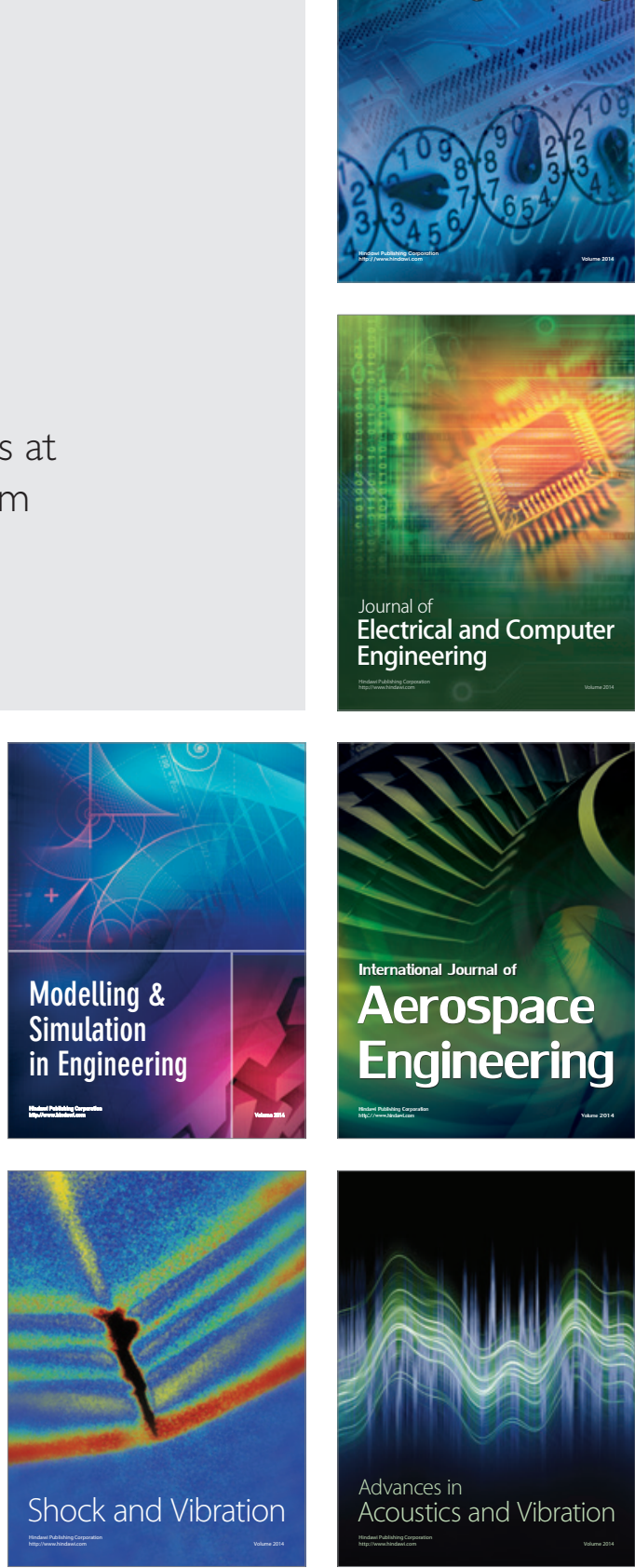